\title{
Optimal Control of Volterra Integrao-Differential Equations: Dickson Interpolation Polynomials and Collocation Method
}

\author{
maryam alipour ${ }^{1}$ and samaneh soradi zeid ${ }^{1}$ \\ ${ }^{1}$ University of Sistan and Baluchestan
}

July 25, 2020

\begin{abstract}
This paper introduces a new direct scheme based on Dickson polynomials and collocation points to solve a class of optimal control problems (OCPs) ruled by Volterra integro-differential equations namely Volterra integro-OCPs (VI-OCPs). Studies in this regard require to calculate the corresponding operational matrices for expanding the solution of this problem in terms of Dickson polynomials. This recommended method allows us to transform the VI-OCP to a system of algebraic equations for choosing the coefficients and control parameters optimally. The error estimation of this technique is also investigated. Finally, some example are given to bring about the validity and applicability of this approach in comparison with those obtained from other methods.
\end{abstract}

\section{Hosted file}

elsarticle-template214.pdf available at https://authorea.com/users/346098/articles/472195optimal-control-of-volterra-integrao-differential-equations-dickson-interpolationpolynomials-and-collocation-method 